\title{
Politique
}

Politique

Générations et politiques sous la direction de Jean Crête et de Pierre Favre, préface de Vincent Lemieux, Québec / Paris, Presses de l'université Laval / Economica, coll. " Politique comparée ", 1989, 370 p.

\section{Bernard Fournier}

Numéro 20, automne 1991

URI : https://id.erudit.org/iderudit/040704ar

DOI : https://doi.org/10.7202/040704ar

Aller au sommaire du numéro

Éditeur(s)

Société québécoise de science politique

ISSN

0711-608X (imprimé)

1918-6584 (numérique)

Découvrir la revue

Citer ce compte rendu

Fournier, B. (1991). Compte rendu de [Générations et politiques sous la direction de Jean Crête et de Pierre Favre, préface de Vincent Lemieux, Québec / Paris, Presses de l'université Laval / Economica, coll. « Politique comparée », 1989, 370 p.] Politique, (20), 163-168.

https://doi.org/10.7202/040704ar d'utilisation que vous pouvez consulter en ligne.

https://apropos.erudit.org/fr/usagers/politique-dutilisation/ 


\section{Générations et politiques}

sous la direction de Jean Crête et de Pierre Favre, préface de Vincent Lemieux, Québec/Paris, Presses de l'université Laval/Economica, coll. «Politique comparée», 1989, 370 p.

Comme le signale d'entrée de jeu l'un des collaborateurs de cet ouvrage collectif, Paolo Giovannini (université de Florencel, le terme "génération" est d'abord un mot du sens commun, largement utilisé dans la vie courante pour décrire diverses réalités familiales, culturelles, artistiques ou politiques. Les auteurs de Acceptation globale n'y faisaient-ils pas référence pour stigmatiser les "40 ans"'? Ne parle-t-on pas de la "génération de la crise", de celle du "baby boom", ou de la «bof-génération"? Préciser cette idée pour en faire un

1. F. Benoit et P. Chauveau, Acceptation globale, Montréal, Boréal, 1986. 
concept scientifique et déterminer sa portée explicative est la tâche à laquelle se sont employés dix chercheurs québécois, canadiens, américains et européens, dans un volume qui fait suite à un colloque tenu à l'université Laval en 1986.

Leurs positions, néanmoins, ne sont nullement univoques, et l'intérêt de cet ouvrage réside justement dans la lecture de démonstrations contradictoires, largement étayées de données d'enquêtes et de tableaux. Des auteurs qui rejettent totalement l'idée de génération au plan scientifique en la reléguant au rang des "facilités de langage» (p. 319) à ceux qui en font un facteur sociologique explicatif au même titre que la classe sociale, sans négliger ceux qui ne la jugent pertinente que dans certains pays et pour certaines époques, le fruit de cette "confrontation scientifique" (p. 1) nourrit le débat et constitue un des seuls ouvrages de synthèse en langue française sur cette question.

Pierre Favre (Institut d'études politiques de Paris) propose même, comme l'une des grilles de lecture possibles du collectif, de suivre ces oppositions. Reprenons l'idée.

$C^{\prime}$ est dans le but d'expliquer certaines situations historiques où s'observent des oppositions réelles en fonction des âges que certains auteurs, tels Richard et Margaret Braungart (Syracuse), croient à l'utilité heuristique du concept de "génération". Ces derniers introduisent utilement le débat en exposant l'historique des études portant sur les générations et les distinctions classiques entre les effets de cycle de vie ll'âge imposerait un type de comportement plutôt qu'un autre), les effets de cohorte (le fait d'être né à peu près au même moment et de vivre des expériences au cours de la jeunesse - que des plus vieux $n^{\prime}$ ont pas vécues - serait déterminant) et les effets de périodes (les circonstances historiques particulières à une époque - guerre, crise, etc. expliqueraient les comportements). $R$. et $M$. Braungart se proposent ensuite d'intégrer ces trois perspectives dans un "modèle interactif des générations politiques», puisqu'il apparaît, à l'analyse, difficile d'en séparer les effets : la conscience politique serait «en grande partie le produit des expériences "personnelles" (cycle de vie) et "sociales" (cohortes) dans un monde qui se transforme (période)" 
(p. 35). Après avoir dégagé quatre «générations politiques» depuis 1815 , les auteurs montrent, entre autres, qu'elles et par conséquent les luttes générationnelles - ne se forment pas par hasard, qu'elles se sont présentées au cours de "périodes extraordinaires", qu'elles ont été menées upar de jeunes leaders charismatiques capables de mobiliser des cohortes de jeunes mécontents pour les rassembler en mouvements actifs œuvrant au changement” (p. 49) et, finalement, que la présence de "générations» n'en cachait pas pour autant les conflits intragénérationnels.

Françoise Ouellet (université Laval), comme les auteurs précédents, insiste aussi sur les deux éléments constitutifs d'une génération politique : la prise de conscience de former un groupe différent en terme d'åge et la mobilisation. Son champ d'investigation, cependant, est plus précis : elle ne travaille que dans le cadre de l'analyse des changements électoraux - un des principaux domaines d'utilisation du concept de génération - et uniquement sur le Québec - où la perspective générationnelle est généralement plus «populaire» qu'ailleurs (p. 184). A partir des sondages préélectoraux et en reprenant le concept de réalignement partisan (crise suivie d'une modification durable des allégeances), elle cherche à vérifier si les réalignements observés sont liés à une prise de conscience et à une mobilisation générationnelle. L'étude des enjeux de chaque élection et les différences observables entre les opinions selon les groupes d'âge lles 1824 ans et les autres) permettent à l'auteure de conclure qu'une unité générationnelle s'est effectivement produite en 1970 en faveur du Parti québécois, unité qui s'est maintenue jusqu'à la victoire de ce parti en 1976. La défection des jeunes électeurs envers ce parti, au milieu des années 80 , confirmait alors que le PO était principalement porté par une même génération.

Dans un autre article portant sur les comportements électoraux, Paul R. Abramson (Michigan State University) se montre cependant plus réservé quant à la portée explicative du concept de génération : le cas américain qu'il analyse n'est d'ailleurs pas propice aux modifications partisanes brutales. De fait, l'auteur s'intéresse plus aux effets du renouvellement 
de la population et à ceux de l'âge qu'à l'idée de génération en tant que prise de conscience et mobilisation. En analysant en détail les cohortes et en éliminant l'effet du renouvellement des générations en stratifiant ses échantillons, Abramson montre que, si le phénomène du changement des générations $n$ 'a pas eu une influence sur la force relative des deux grands partis, il s'avère un facteur important de l'affaiblissement global de l'identification partisane aux États-Unis (les jeunes électeurs s'identifiant de moins en moins à un parti, et cette absence d'identification étant constante au cours de leur vie [p. 128]. Pour Abramson, si l'impact des effets de génération peut être discuté, le fait qu'il existe des cohortes politiquement distinctes reste plus aisément démontrable (p. 122).

Richard Johnston (université de Colombie-Britannique), quant à lui, ne croit pas que les déplacement électoraux au Canada s'expliquent en fonction d'une logique de l'âge. Tout en reconnaissant cependant une certaine particularité à la situation québécoise, il lui semble que les changements à long terme résultent généralement d'une conversion des électeurs quel que soit leur âge, et non d'un renouvellement propre a l'effet de génération (p. 152). La faiblesse des bases sociales des partis politiques canadiens expliquerait cette fragilité des engagements partisans et la difficulté de réfléchir en termes de génération : les électeurs canadiens sanctionnent davantage un parti qu'ils ne l'approuvent, et cette sanction peut être faite à tout åge (p. 178). John Curtice (université de Strathelyde), analysant la situation en Grande-Bretagne, en arrive sensiblement aux mêmes conclusions - contrairement à de nombreux auteurs qui identifient aussi les changements politiques britanniques à des phénomènes de génération (p. 236).

On remarquera, néanmoins, que ces auteurs plus critiques analysent surtout les «effets générationnels" en travaillant sur l'impact discriminant de l'âge (soit dans le déplacement des orientations partisanes en fonction de l'åge ou, au contraire, sur la persistance pour une même cohorte et les oppositions aux autres). II s'agit certainement d'une réduction importante de l'idée de génération, qui n'est plus considérée comme une prise de conscience et une mobilisation. II en va de même en 
limitant l'étude au seul niveau électoral, ce que Curtice reconnaît en concluant son article : si des effets générationnels peuvent exister au niveau des valeurs et des expériences, "leur traduction électorale demeurera faible» (p. 253). Bien que Paolo Giovannini adopte une perspective plus large dans son article sur les jeunes Italiens et leur rapport à la politique, il n'en reste pas moins que l'auteur insiste plus sur l'impact de l'entrée des nouvelles cohortes - qui se révèle d'ailleurs important - que sur celui de la mobilisation générationnelle.

II ne faut pas alors s'étonner que la pertinence des découpages en fonction de l'åge devienne un des points d'appui de la critique de Pierre Favre. Si le seul indicateur de l'âge sert au découpage de générations ou de cohortes, le problème de l'homogénéité sociologique de ces regroupements se pose : selon les contextes, les individus appartiennent à plusieurs "générations" et chaque champ social possède sa dynamique propre. La portée heuristique du concept s'en trouve fortement limitée (p. 288). Même en dépassant ces premières critiques (qui, selon nous, s'appliquent d'ailleurs à plusieurs autres concepts sociologiques), le chercheur se voit toujours limité dans la construction même de son corpus, puisque l'état actuel de ses instruments d'analyse ne permet pas de "caractériser avec un minimum de précision des générations successives" (p. 304). L'objection est connue : R. et $M$. Braungart ne signalent-ils pas que des données quantitatives sur plus de 100 ans seraient nécessaires? Pis encore, ces dernières obtenues, rien n'assure que des comparaisons sur de si longues périodes soient possibles, les questions posées en 1991 ayant toutes les chances de ne plus être pertinentes en 2091. C'est dans ce contexte, à notre avis, que I'article de André Blais et Jean Crête prend tout son sens. Ces auteurs tentent en effet de construire de tels instruments de comparaison en évaluant les changements politiques sur une trentaine d'années, dans dix-huit démocraties capitalistes avancées, en travaillant non pas à partir de quelque typologie à priori, mais à partir des données disponibles. L'exercice montre cependant que les seules propositions comparables et qui ont été retenues ne peuvent qu'être larges, et perdent sensiblement, de ce fait, leur intérêt. 
Comme on le voit, ce livre est riche d'enseignements et constitue lui-même une démarche critique essentielle aux chercheurs intéressés par ces questions. On remarquera seulement que certaines positions $n$ ' apparaissent contradictoires que parce qu'elles reposent sur des interprétations divergentes du concept de génération, que des critiques sur l'homogénéité des cohortes sont pertinentes s'il s'agit de ne comparer que les comportements électoraux en fonction de classes d'åge, mais qu'elles le deviennent moins si la génération est envisagée comme un groupe conscient de son unité générationnelle et mobilisé dans le but d'une action. En ce sens, la génération qui porta le Parti québécois et que Ouellet - et d'autres chercheurs - ont identifiée en est peut-être le meilleur modèle.

II faut regretter toutefois que le livre $n$ 'ait pas fait l'objet d'une révision plus minutieuse : l'orthographe des noms propres peut varier dans une même page (cf. p. 67), et les coquilles et fautes sont nombreuses. Cela ne peut que nuire a une lecture par ailleurs fort enrichissante.

Bernard Fournier Institut d'études politiques de Paris 